\title{
Clear speech perception in specific language impairment and delayed language affecting phonology children
}

\begin{abstract}
Purpose: To evaluate the performance of children with specific language impairment (SLI) and delayed language affecting phonology on Arabic clear speech.

Method: This prospective case-control study included 30 children suffering from SLI and delayed language affecting phonology with multiple phonological processes and 30 control children. Both groups were evaluated for their performance on the standardized Arabic clear speech test material. All participants have been assigned randomly to the study from the Phoniatrics outpatient clinic. Results were compared between the two groups.

Results: A significant clear speech advantage was observed in intelligibility for all participants. Clear speech advantage in children with SLI and delayed language affecting phonology was $32 \%$ over conversational speech $(\mathrm{P}=0.002)$.

Conclusion: Children with SLI and delayed language affecting phonology performed better on clear speech than on conversational speech. This study suggests the use of clear speech in evaluation and remediation of children with SLI and delayed language affecting phonology in order to correct their phonological processes and improve their ability to communicate.
\end{abstract}

Keywords: clear speech, speech perception, delayed language affecting phonology, sli
Volume 9 Issue I - 2017

\author{
Salwa Abd El Mawgouda, Ahmed Aboudb , \\ Megahed M Hassanc \\ Otolaryngology Department Faculty of Medicine Sohag \\ University, Egypt
}

Correspondence: Salwa Abd El Mawgouda Audiology Unit Otolaryngology Department Faculty of Medicine Sohag University Sohag, Egypt, Tel +2 (0) | 285395808 , Emailsalwa_mamgoud@yahoo.com

Received: June 14,2017 | Published: November 07, 2017

\section{Introduction}

A child with a phonological delay/disorder has difficulty producing speech. Those are likely to have difficulties with all aspects of phonological awareness including discriminating between sounds, holding several sounds in their short-term memories and blending sounds. Both real and pseudo words will be affected. ${ }^{1}$ Phonological disorder will involve some delay, but also the use of phonological processes that are atypical, inconsistent or not following the expected pattern of phonological development. This is likely to make the child less clear. ${ }^{2}$ Tallal's temporal processing theory of SLI explained the problems experienced by children with SLI as arising from a difficulty integrating sensory stimuli that converges in rapid succession in the central nervous system (if these stimuli were presented at fast pace or if they were brief in duration). ${ }^{3}$

Children with phonological difficulties may need more time to process and produce their responses. ${ }^{4}$ Children with phonological difficulties will be helped by any visual approaches and programs that allow staff and child to refer to sounds through gesture or sign. They will also benefit from color coded systems as visual reminders of language structures or of sound groups. Awareness of their own speech sounds and language abilities (Metaphonic and Metalinguistic awareness) are also essential; ensuring the child has the necessary concepts and vocabulary to discuss these. ${ }^{2}$

One effective means of improving speech intelligibility is to speak clearly. The higher intelligibility in clear speech than in conversational speech is likely a result of acoustic and phonetic differences between these two styles of speech. ${ }^{5}$ The benefit has been demonstrated in diverse populations including those with learning disabilities, auditory neuropathy, and cochlear implants. ${ }^{6}$ Temporal deficit is a hallmark of delayed language affecting phonology ${ }^{7}$ and SLI. ${ }^{3}$ The current research was dedicated to answer the question (is the temporal modification of speech beneficial for better speech understanding in those children?). So, the purpose of this work is to evaluate the performance of children with SLI and delayed language affecting phonology on Standardized Arabic clear speech test material ${ }^{8}$ and to compare their performance with that of normal children.

\section{Methods}

\section{Participants}

The present study was conducted in Sohag University Hospital, Egypt in the period from May 2016 to February 2017. It consisted of two groups; control group included 30 children with the following inclusion criteria: Age range from 3-12years. Gender: males and females. No history of delayed language development. Normal hearing sensitivity not exceeding $15 \mathrm{dBHL}$ in the frequencies from $250 \mathrm{~Hz}$ to $8000 \mathrm{~Hz}$ by air conduction. Normal word discrimination score. Study group: consists of 30 children with delayed language affecting phonology and SLI with age range from 3-12years. Delayed language affecting phonology children $(n=12)$ have the following inclusion criteria: Diagnosis was established as delayed language affecting phonology according to standard protocol in Phoniatrics Unit. That is language test revealed normal semantic and syntax development for age; articulation test showed phonological processes or delay of three sounds or more. Sounds affected must belong to 3 different groups of distinctive features. Intelligent quotient (I.Q) is normal or below average. The SLI children $(\mathrm{n}=18)$ were diagnosed according to our protocol as phonologic-syntactic type. That is having positive family history, normal I.Q, inconsistent phonologic errors, grouping, consonants are more affected than vowels. Language test showed delayed semantic, syntax and phonology for age. All children did not undergo language therapy or any management method previously. 


\section{Speech material}

The stimuli used included 144 speech sentences recorded in clear and conversational speech styles. Speech sentences were separated into 18 lists, each containing 8 sentences and with 25 key words in each list (Appendix $1 \& 2$ ). The first sentence in each list has four key words, and the remaining sentences each have three.

Criteria of clear speech sentences: a) Slower speaking rate (the duration was double that of conversational sentences), b) more and longer pauses, c) increased energy in the 1000-3000 Hz range, d) targeted vowel formants, e) increased consonant intensity compared to adjacent vowels (more stress on consonant).

\section{Procedure}

Ethical committee approved this work. Written consent was taken from all participants' parents. All children were tested in a doublewalled, sound-treated room. Each child in the two groups was subjected to the following: The lists were presented at the most comfortable loudness level. To familiarize the children with the test materials and procedures, a short session with 3 sentences were conducted at the beginning of the test. All children were presented with stimuli via headphone and the child is asked to repeat in 2 conditions; condition No. I: conversational speech sentences and condition No. II: clear speech sentences.

In scoring and data collection, the examiner recorded the score for the correct key words in each sentence. Give one point for each key word repeated correctly by the child (key words are under- lined on the score sheets).

\section{Statistics}

Paired t-test was used to compare the performance on the conversational versus clear speech sentences in each group. Also, the unpaired t-test was used to compare both conversational and clear speech performances between the two groups. The $\mathrm{P}$ values were twotailed with confidence intervals $95 \%$.

\section{Results}

Mean age for normal children in the control group was 9years, while it was $8.5 y$ years for children with delayed language affecting phonology and SLI. The control group includes 14 (46.67\%) normal females and $16(53.33 \%)$ normal males. The gender distribution in children with delayed language affecting phonology and SLI was $20(66.67 \%)$ males and $10(33.33 \%)$ females. Results showed no significant difference between performance of the control group on conversational and clear speech conditions $(\mathrm{P}=0.072$; Table I). In contrast, children with SLI and delayed language affecting phonology performed much better on clear speech than on conversational speech with significant difference ( $\mathrm{P}=0.002$; Table II). Comparing the conversational speech performances between the two groups revealed highly significant difference $(\mathrm{P}<0.001)$ and $\mathrm{t}$ value 10.42 . Similarly, the clear speech performances showed significant difference between the two groups $(\mathrm{P}<0.001)$ with $\mathrm{t}$ value 5.99 (Figure 1).

\section{Discussion}

The authors compared perception of conversational versus clear speech in normal hearing children. In the present study the authors used clear speech in slow rate which means that at a longer duration than conversational speech (clear/slow). There was no statistically significant difference between the performances in both conditions in normal children as the mean values were 96 and 100 respectively.
Though, clear speech scores are slightly better than conversational speech (Table 1). These results agree with the study done by ${ }^{9}$ on normal hearing subjects. Their study showed that clear speech at normal rates (same duration of conversational speech) was more intelligible than conversational speech and was almost as beneficial as clear speech at slow rates (clear/slow).

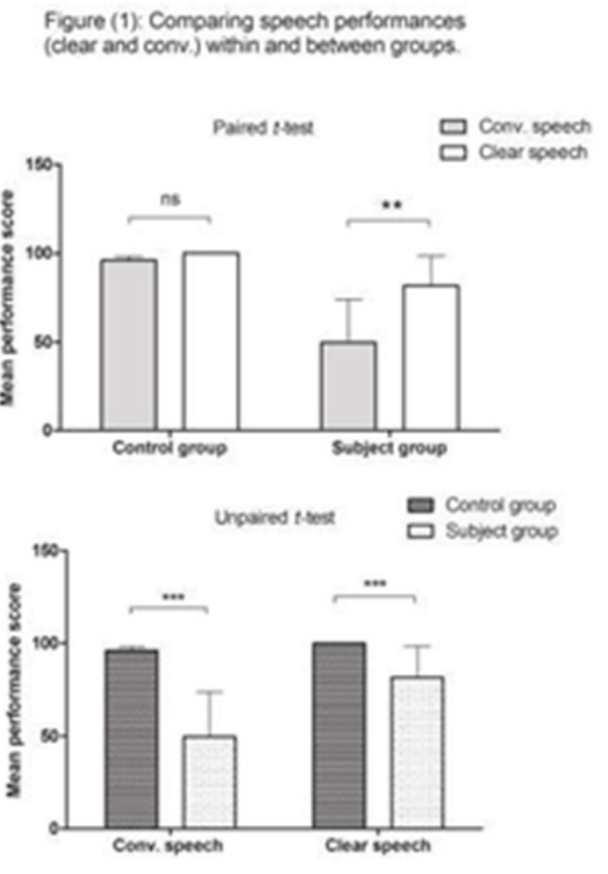

Figure I Comparison of speech performances within and between groups (subject and control).

Results of paired and unpaired $t$-tests.

Abbreviations: Conv: Conversational; NS: Non-Significant; $* * P<0.01$; ***P $<$ $0.00 \mathrm{I}$.

Table I Results of the 2 conditions in the control group (Mean, SD and Range)

\begin{tabular}{llll}
\hline Condition & Mean & SD & Range \\
\hline Condition I (Conversational speech) & 96 & 2.16 & $88-100$ \\
Condition II (Clear Speech) & 100 & 0 & $100-100$ \\
\hline
\end{tabular}

The $\mathrm{P}$-value is 0.072 which is not significant at $\mathrm{P}<0.05$

Evaluation of the performance of children with delayed language affecting phonology and SLI on Arabic clear speech demonstrates a significant clear speech advantage. The mean value of conversational speech is $49.6 \%$ while it is $81.6 \%$ in clear speech condition with an advantage equals to $32 \%$ for clear over conversational speech (Table 2). Perception of clear speech than conversational speech in children with delayed language affecting phonology and SLI can be explained by some factors. One of these factors is that clear speech is characterized by greater temporal amplitude modulations than conversational speech. Because delayed language affecting phonology as well as SLI may be attributed to a temporal processing deficit, the enhanced temporal properties in clear speech could be beneficial to those children. Other factor is that clear speech production has the effect of acoustic-phonetic modification of speech signals. A wellknown previous somewhat similar method was used in remediation; the auditory bombardment. ${ }^{10}$ Advocated using auditory bombardment to facilitate phonological development. They argued that phonological development is acquired by listening. Auditory bombardment might 
consist of listening to a story that contains numerous examples of target sounds. However, auditory bombardment did not fulfill clear speech characteristics. Distinctiveness between phonological categories is enhanced in clear speech compared with conversational speech.

Table 2 Results of the 2 conditions in the study group (Mean, SD and Range)

\begin{tabular}{llll}
\hline Condition & Mean & SD & Range \\
\hline Condition I (Conversational Speech) & 49.6 & 24.29 & $20-88$ \\
Condition II (Clear Speech) & 81.6 & 16.8 & $72-100$ \\
\hline
\end{tabular}

The $\mathrm{P}$-value is 0.002 which is significant at $\mathrm{p}<0.05$

\section{Recommendations}

Our study recommended the use of standardized clear speech material in evaluation test battery for delayed language affecting phonology and SLI children. In addition, we recommend the application of clear speech in remediation of those children in order to improve their ability to communicate.

\section{Conflicts of interest}

Author declares there are no conflicts of interest.

\section{Acknowledgments}

None.

\section{Funding}

None.

\section{References}

1. Joffe V, Pring T. Children with phonological problems: A survey of clinical practice. Int J Lang Commun Disord. 2008;43(2):154-164.
2. Dodd B. Children with speech disorder. Defining the problem. In: Dodd B (Ed.), Differential diagnosis and treatment of children with speech disorders, West Sussex, Whurr Publishers, UK. 2005. p.3-23.

3. Tallal P, Miller S, Fitch RH. Neurobiological basis of speech: a case for the preeminence of temporal processing. In: Tallal P, et al. (Eds.), Temporal information processing in the nervous system: Special reference to dyslexia and dysphasia. New York: Academy of Sciences, New York, USA. 1993. p.27-47.

4. Wagner R, Torgesen J, Laughon P, et al. Development of young reader's phonological processing abilities: New evidence in bi-directional causality from a latent variable longitudinal study. Developmental Psychology. 1993;30(1):73-87.

5. Liu S, Del Rio E, Bradlow A, et al. Clear speech perception in acoustic and electric hearing. Journal of the Acoustical Society of America. 2004;116(4):2374-2383

6. Bradlow A, Kraus N, Hayes E. Speaking clearly for children with learning disabilities: Sentence perception in noise. J Speech Lang Hear Res. 2003;46(1):80-97.

7. Soares AJ, Sanches SG, Alves DC, et al. Temporal auditory processing and phonological awareness in reading and writing disorders: preliminary data. Codas. 2013;25(2):188-190.

8. Mourad S. Clear Speech Perception in Patients with Auditory Neuropathy. Egyptian Journal of Ear, Nose, Throat and Allied Sciences. 2016.

9. Krause J, Braida L. Investigating alternative forms of clear speech: The effects of speaking rate and speaking mode on intelligibility. $J$ Acoust Soc Am. 2012;112(5 Pt 1):2165-2172.

10. Hodson BW, Paden EP.Targeting intelligible speech (2nd edn), Austin, T.X: Pro-Ed, USA. 1991. 